\title{
Mechanism of Youguiyin In The Treatment of Osteoporosis Based On Network Pharmacology And Molecular Docking
}

\section{Yi Pan}

Zhejiang Chinese Medical University https://orcid.org/0000-0001-7911-7962

\section{Wanlu Zhao}

Zhejiang Chinese Medical University

\section{Luping Qin}

Zhejiang Chinese Medical University

Lu Zhang ( $D$ 20181020@zcmu.edu.cn )

Zhejiang Chinese Medical University https://orcid.org/0000-0002-1477-6104

\section{Research}

Keywords: Youguiyin, Osteoporosis, Network pharmacology, Molecular docking, Mechanism

Posted Date: September 28th, 2021

DOI: https://doi.org/10.21203/rs.3.rs-798949/v2

License: (c) (i) This work is licensed under a Creative Commons Attribution 4.0 International License. Read Full License 


\section{Abstract}

Background: Youguiyin (YGY) has been confirmed to treat osteoporosis (OP) in clinical trials, but its specific pharmacological mechanism remains unclear. This study aimed to explore the material basis and potential mechanism of YGY in the treatment of OP based on network pharmacology and molecular docking.

Methods: Databases including TCMSP, SwissTargetPrediction database, OMIM, and TTD were used to predict the effective ingredients and relevant targets of YGY in the treatment of OP. The STRING database was used to reveal the relationship between each intersection target protein. Metascape database was used to perform GO enrichment analysis and KEGG pathway enrichment analysis on the intersection targets. Cytoscape 3.6.0 software was used to show the complex network relationship of YGY in the treatment of OP. According to the results of network characteristics analysis, the core effective ingredients and the core targets were screened out. Autodock 4.0 was used for molecular docking and Pymol was used to visualize the docking results.

Results: 290 effective ingredients, 1127 targets of the effective ingredients, 273 relevant targets of OP and 17 intersection targets were screened out in total by searching literature and databases. Intersection targets could affect biological processes including regulation of inflammatory response, ossification, negative regulation of post-transcriptional gene silencing, positive regulation of cytokine biosynthetic process and regulation of hormone levels by regulating signal pathways including TNF signaling pathway, osteoclast differentiation, apoptosis, MAPK signaling pathway and PI3K/Akt signaling pathway. Through screening, 14 core effective ingredients and 6 core targets were confirmed. The results of molecular docking showed that most of the core effective ingredients including a-humulene, cinnamaldehyde, denudatine, benzoylhypaconine and quercetin had good binding activity with the core targets including TNF- $a$, IL-1 $\beta$ and IL-6.

Conclusion: Based on network pharmacology and molecular docking, the critical effective ingredients, key targets, important signal pathways and main biological processes of YGY in the treatment of OP were successfully screened out. This study revealed the material basis and the mechanism of YGY in the treatment of OP and provided a theoretical basis for follow-up experimental research and clinical application of YGY.

\section{Introduction}

Osteoporosis (OP) is a kind of systemic metabolic skeletal disease mainly characterized by the decrease of bone tissue content per unit volume and the destruction of bone tissue microstructure, which lead to the increase of bone fragility and risks of fracture. With the prolongation of human life expectancy and the aging of the population, OP has aroused widespread concern in society. The OP epidemiological survey report released by China in 2018 [1] showed that the prevalence rate of people aged from 40 to 49 was $3.2 \%$, the prevalence rate of people over 50 years old was $19.2 \%$, and the prevalence rate of people 
over 65 years old was $32.0 \%$. The prevalence of osteoporosis is increasing year by year [2]. It is predicted that by 2050, the number of fractures caused by osteoporosis in China will reach 5.99 million, which will seriously affect people's quality of life [3]. The current prevention and treatment methods of OP are mainly limited to bone resorption-inhibiting therapy such as bisphosphonates, selective estrogen receptor modulator SERM and calcitonin, and bone formation-promoting therapy such as parathyroid analogs, teriparatide [4]. These methods have good prevention and treatment effects, but some serious adverse reactions can't be avoided, limiting their clinical applications. Traditional Chinese medicine has fewer side effects. A large number of clinical studies have confirmed that traditional Chinese medicine compounds and proprietary Chinese medicine can improve the clinical symptoms of OP with good therapeutic effect. Traditional Chinese medicine therapy is a very promising direction of OP treatment, However, due to the complex mechanism of traditional Chinese medicine compounds in the treatment of diseases, the development of traditional Chinese medicine therapy is restricted to a certain extent. Therefore, the mechanism of traditional Chinese medicine needs to be further studied.

"Huang Di Nei Jing" considers that the deficiency of kidney-yang is the root cause of OP [5]. Combined with modern clinical research, OP mostly occurs in elderly population especially in postmenopausal women [1]. Most patients with OP have symptoms of kidney-yang deficiency such as aches of waist and knee and cold limbs [6]. Therefore, tonifying the kidney-yang is one of the important parts in the treatment of OP.

Youguiyin (YGY) is a classic prescription for treating kidney-yang deficiency, which was recorded in Jing Yue Quanshu written by Jiebing Zhang in Ming Dynasty. This prescription is composed of Cortex cinnamomi (Rougui, RG), Radix Aconiti lateralis praeparata (Fuzi, FZ), Radix rehmanniae praparata (Shudi, SD), Cornus officinalis (Shanzhuyu, SZY), Lycii Fructus (Gouqizi, GQZ), Rhizoma Dioscoreae (Shanyao, SY), Eucommia ulmoides (Duzhong, DZ) and Glycyrrhiza uralensis (Gancao, GC). YGY is mainly used to treat cold limbs, aches of waist and knee, and other symptoms which are common in OP patients of kidney-yang deficiency type. Recent research shows that YGY has significant effects in the treatment of OP clinically. It can effectively promote the proliferation and differentiation of osteoblasts (OB) to prevent its apoptosis, and meanwhile it can inhibit the formation and differentiation of osteoclasts (OC) to promote its apoptosis [7, 8]. However, the specific pharmacological mechanisms and molecular targets of YGY have not been clarified, which limits the promotion and application of this prescription. Therefore, the monarch and minister medicines (RG, FZ, SD, SZY, GQZ, SY) that play the role of tonifying kidney-yang in YGY were selected for this study.

The multiple effective ingredients in traditional Chinese medicine compounds can exert different medicinal effects against different targets, forming an extremely complex drug-component-target network [9]. Network pharmacology is a scientific discipline that studies the interaction between drugs and the body based on the perspective of biological networks to discover new drugs [10]. This method of drug discovery is more in line with the overall concept of traditional Chinese medicine theory because it is not limited to the "single-component,single-target,single-pathway" model, and it also reduces the failure risk and economic loss of drug development to a greater extent. 
Therefore, network pharmacology method was used to explore the potential mechanism of YGY in the treatment of OP. The effective ingredients of YGY and the common targets related to YGY and OP were selected to construct a Chinese medicine-compound-disease-target network. The binding ability between the core effective ingredients and core targets was evaluated through molecular docking to further elaborate the mechanism of YGY at the molecular level. The results of this study may help to clarify the mechanism of YGY in the treatment of OP and provide a theoretical reference for its clinical application. The flow chart of this study is shown in Fig. 1.

\section{Materials And Methods}

\subsection{Acquisition of YGY's effective ingredients}

The compounds and the corresponding ADME parameters of RG, FZ, SD, SZY, GQZ and SY were collected by the Traditional Chinese Medicine Systems Pharmacology Database and Analysis Platform (TCMSP, https://tcmspw.com/tcmsp.php) [11]. Compounds with oral bioavailability $\geq 30 \%$, durg-likeness $\geq 0.18$ and half-life $\geq 4 \mathrm{~h}$ were screened as effective ingredients. At the same time, the main effective ingredients of the six Chinese medicine in relevant literature were consulted and merged into the screening results.

\subsection{Acquisition of the targets involved in YGY and OP}

The targets of the collected effective ingredients were retrieved from TCMSP and the Swiss Target Prediction database (http://www.swisstargetprediction.ch/index.php) [12]. The OP-related targets were provided by Therapeutic Target Database (TTD,http://db.idrblab.net/ttd/) [13] and OMIM (https://www.omim.org) [14]. With the help of David (https://david.ncifcrf.gov/) [15], protein names of all targets were converted to the corresponding gene symbol for further screening.

2.3 Acquisition of potential targets of YGY in the treatment of OP

The intersection targets of YGY and OP were selected as the potential targets of YGY in the treatment of $\mathrm{OP}$, and a Venn diagram was constructed at the same time.

\subsection{Construction of the Chinese medicine-compound-OP-target network}

The Chinese medicine-compound-OP-target network was constructed by Cytoscape 3.6.0 software according to the relationships of the intersection targets, the effective ingredients and Chinese medicinal materials. The degree of each effective ingredient and each intersection target from the analysis of network characteristics could be used as one of the basis for screening core effective ingredients and core targets.

\subsection{Construction of the protein-protein interaction (PPI) network}

The intersection targets were imported into the STRING database (https://string-db.org/) [16] to obtain the interactions between proteins by setting the species as "Homo sapiens" and the minimum interaction 
threshold at 0.400. Furthermore, the "Network Analyze" tool in Cytoscape 3.6.0 software was used to perform the analysis of network characteristics and the construction of PPI network. Similarly, the degree of each intersection target from the analysis could be used as one of the basis for screening core targets.

\subsection{GO enrichment analysis and KEGG pathway enrichment analysis}

The intersection targets were imported into the Metascape database (http://metascape.org/) [17] for GO enrichment analysis and KEGG pathway enrichment analysis to explore the gene functions and signal pathways involved. The results of the above analysis were illustrated with bar chart and bubble chart respectively. In addition, the relationship between the intersection targets and the top 20 signal pathways was imported into Cytoscape 3.6.0 software to construct the intersection target-signal pathway network. Similarly, the degree of each intersection target from the analysis of network characteristics could be used as one of the basis for screening core targets.

\subsection{Molecular docking}

The core targets were screened out based on the degree of each intersection target obtained by the above 3 network characteristics analyses. And the protein structures of the core targets in pdb format were obtained from the RCSB Protein Data Bank (https://www.rcsb.org/) [18]. Correspondingly, the core effective ingredients were determined based on the degree of each effective ingredient and relevant literature. And the sdf files of the core effective ingredients downloaded from the Pubchem database (https://pubchem.ncbi.nlm.nih.gov/) [19] were converted into mol2 files by Open Babel. The core target protein and core active ingredients processed by AutoDock Tools 1.5.6 software were saved as pdbqt files for molecular docking in AutoDock The binding energy was calculated by AutoDock-Vina to evaluate the binding ability between the targets and the effective ingredients. Generally speaking, the binding activity between the component and the target is considered great if their binding energy is less than -4.25 ; excellent if their binding energy is less than -5.00 , and intense if their binding energy is less than -7.00 [20]. In addition, Pymol was utilized to visualize the results of molecular docking, so as to more intuitively explain the mechanism of YGY in the treatment of OP at the molecular level.

\section{Results}

\subsection{The effective ingredients and targets of YGY}

Through the retrieval of literature and databases, the effective ingredients and relevant targets of YGY were selected, including 100 effective ingredients of RG, 64 effective ingredients of $F Z, 74$ effective ingredients of SD, 16 effective ingredients of SZY, 42 effective ingredients of GQZ, 16 effective ingredients of SY, 443 targets of RG, 570 targets of FZ, 526 targets of SD, 70 targets of SZY, 208 targets of GQZ, and 80 targets of SY.

3.2 The relevant targets of $\mathrm{OP}$ and the intersection targets of $\mathrm{YGY}$ and $\mathrm{OP}$ 
273 relevant targets of OP were collected from TTD and OMIM with the keyword "osteoporosis". After the construction of the Venn diagram (Fig. 2), 17 intersection targets of YGY and OP were selected as the potential targets of YGY in the treatment of OP.

\subsection{The Chinese medicine-compound-OP-target network}

The relationships of the intersection targets, the effective ingredients and Chinese medicinal materials was imported into Cytoscape 3.6.0 software to construct the Chinese medicine-compound-OP-target network (Fig. 3). In this network, 42 ingredients from 6 Chinese medicines were able to act on 17 targets, which reflected the characteristics of Chinese medicine compound as multi-component and multi-target. The result of network characteristics analysis showed that the effective ingredients with higher degree were mainly distributed in RG, FZ and SD, including FZ6 (denudatine), FZ2 (2,7-dideacetyl-2,7-dibenzoyltaxayunnanine F), RG9 (a-humulene), RG15 (palmitic acid), A1 (quercetin) and SD9 (aucubin); while the targets with higher degree mainly included CASP3, CNR2, ESR1, TNF and so on. The effective ingredients and targets with top 10 degree were shown in Table 1 and Table 2, which could be used as the basis for screening core effective ingredients and core targets.

Table 1

The effective ingredients with top 10 drgee in the Chinese medicine-compound-OP-target network

\begin{tabular}{|llll|}
\hline Serial number & Compound name & Degree & Source \\
\hline A1 & Quercetin & 7 & SD, RG, GQZ \\
\hline SD9 & Aucubin & 2 & SD \\
\hline RG15 & Palmitic acid & 2 & RG \\
\hline RG9 & Alpha-humulene & 2 & RG \\
\hline FZ6 & Denudatine & 2 & FZ \\
\hline FZ2 & 2,7-Dideacetyl-2,7-dibenzoyl-Taxayunnanine F & 2 & FZ \\
\hline B1 & Beta-sitosterol & 1 & GQZ, SZY \\
\hline SY2 & hancinone C & 1 & SY \\
\hline SY1 & Garcinone-B & 1 & SY \\
\hline SD13 & Uridine & 1 & SD \\
\hline
\end{tabular}


Table 2

The targets with top 10 drgee in the Chinese medicine-compound-OP-target network

\begin{tabular}{|lll|}
\hline Gene symbol & Target name & Degree \\
\hline CASP3 & Caspase-3 & 9 \\
\hline CNR2 & Cannabinoid receptor 2 & 7 \\
\hline ESR1 & Estrogen receptor & 7 \\
\hline TNF & Tumor necrosis factor & 5 \\
\hline TERT & Telomerase reverse transcriptase & 5 \\
\hline CTSK & Cathepsin K & 5 \\
\hline IL6 & Interleukin-6 & 3 \\
\hline COL1A1 & Collagen alpha-1(I) chain & 3 \\
\hline IL1B & Interleukin-1 beta & 2 \\
\hline ALOX15 & Polyunsaturated fatty acid lipoxygenase ALOX15 & 2 \\
\hline
\end{tabular}

\subsection{The PPI network}

The results of protein interaction relationship obtained from the STRING database was imported into Cytoscape 3.6.0 software to construct the PPI network (Fig. 4). This network showed the interaction of 16 targets, and the depth of the color indicated the degree of each target and the strength of the interaction.. The result of network characteristics analysis showed that the targets with higher degree including IL-6, TNF, IL-1 $\beta$, SPP1 and CASP3 may play an important role in the treatment of OP by YGY. The targets with top 10 degree were shown in Table 3, which could be used as the basis for screening core targets. 
Table 3

The targets with top 10 drgee in PPI network

\begin{tabular}{|lll|}
\hline Gene symbol & Target name & Degree \\
\hline IL6 & Interleukin-6 & 14 \\
\hline TNF & Tumor necrosis factor & 12 \\
\hline IL1B & Interleukin-1 beta & 12 \\
\hline SPP1 & Osteopontin & 10 \\
\hline CASP3 & Caspase-3 & 10 \\
\hline ESR1 & Estrogen receptor & 9 \\
\hline RUNX2 & Runt-related transcription factor 2 & 8 \\
\hline IL4 & Interleukin-4 & 8 \\
\hline NFKB1 & Nuclear factor NF-kappa-B p105 subunit & 7 \\
\hline COL1A1 & Collagen alpha-1(I) chain & 7 \\
\hline
\end{tabular}

3.5 GO enrichment analysis and KEGG pathway enrichment analysis

17 intersection targets were imported into the Metascape database for GO enrichment analysis and KEGG pathway enrichment analysis. There are 482 biological processes, 11 cell components, 10 molecular functions and 54 signal pathways involved in 17 intersection targets. Obviously, YGY exerts its therapeutic effects by affecting multiple biological functions and multiple signal pathways. The top 10 biological processes, cell components and molecular functions, and the top 20 signal pathways were screened out based on the logP value. Additionally, the results were represented in the form of bar chart (Fig. 5) and bubble chart (Fig. 6).

It can be seen from Fig. 5 that the biological processes involved in the intersection target mainly included negative regulation of post-transcriptional gene silencing, regulation of inflammatory response, positive regulation of cytokine biosynthetic process, regulation of monooxygenase activity, regulation of hormone levels and ossification; the cell components involved mainly included side of membrane, cytoplasmic side of plasma membrane, extrinsic component of plasma membrane, dendrite and neuronal cell body; the molecular functions involved mainly included cytokine receptor binding, cytokine activity, receptor ligand activity, receptor regulator activity and growth factor receptor binding.

As shown in Fig. 6, the signal pathways involved in the intersection targets mainly included IL-17 signaling pathway, Toll-like receptor signaling pathway, TNF signaling pathway, osteoclast differentiation, MAPK signaling pathway, apoptosis, PI3K/Akt signaling pathway and NOD-like receptor signaling pathway. 
Based on the targets enriched in the top 20 signal pathways, Cytoscape 3.6 .0 software was used to construct the intersection target-signal pathway network (Fig. 7). According to the results of network characteristics analysis, the targets with high degree value including NF-kB, IL-6, IL-1 3, TNF and CASP3 may be the key factors of YGY in the treatment of OP. The targets with top 10 degree were shown in Table 4 , which could be used as the basis for screening core targets.

Table 4

The targets with top 10 drgee in the intersection target-signal pathway network

\begin{tabular}{|llc|}
\hline Gene symbol & Target name & Degree \\
\hline NFKB1 & Nuclear factor NF-kappa-B p105 subunit & 18 \\
\hline IL6 & Interleukin-6 & 17 \\
\hline IL1B & Interleukin-1 beta & 17 \\
\hline TNF & Tumor necrosis factor & 16 \\
\hline CASP3 & Caspase-3 & 11 \\
\hline IL4 & Interleukin-4 & 8 \\
\hline CTSK & Cathepsin K & 4 \\
\hline PPP3CA & Serine/threonine-protein phosphatase 2B catalytic subunit alpha isoform & 4 \\
\hline COL1A1 & Collagen alpha-1(I) chain & 2 \\
\hline SPP1 & Osteopontin & 2 \\
\hline
\end{tabular}

\subsection{Molecular docking}

Based on Table 1 and relevant literature [21-23], 14 core effective ingredients were determined (Table 5). Considering the ranking of each target in Table 2, Table 3, and Table 4, the following core targets were screened out: TNF, CASP3, CTSK, IL-1 $\beta$, NF-kB and IL-6. The docking results of the core effective ingredients and the core targets were shown in Fig. 8 and Fig. 9. As shown in the docking results, ahumulene, cinnamaldehyde and denudatine were able to bind tightly to all core targets with the binding energy less than -7.00 . Meanwhile, the binding energy between IL-1 $\beta$ and 14 core effective ingredients were all less than -5.00 . Most of the effective ingredients showed good binding activity to the core targets, which validated the therapeutic effect of YGY on OP. 
Table 5

Information of core effective ingredients

\begin{tabular}{|lll|}
\hline Serial number & Compound name & Source \\
\hline A1 & Quercetin & SD, RG, GQZ \\
\hline SD9 & Aucubin & SD \\
\hline RG15 & Palmitic acid & RG \\
\hline RG9 & Alpha-humulene & RG \\
\hline FZ6 & Denudatine & FZ \\
\hline FZ2 & 2,7-Dideacetyl-2,7-dibenzoyl-Taxayunnanine F & FZ \\
\hline N/A & Benzoylaconine & FZ \\
\hline N/A & Benzoylmesaconine & FZ \\
\hline N/A & Benzoylhypaconine & FZ \\
\hline N/A & Aconitine & FZ \\
\hline N/A & Mesaconitine & FZ \\
\hline N/A & Hypaconitine & $F Z$ \\
\hline N/A & Cinnamaldehyde & RG \\
\hline N/A & Catalpol & SD \\
\hline
\end{tabular}

\section{Discussion}

It is generally believed that $\mathrm{OP}$ is closely related to the negative balance of bone resorption and bone formation [24]. Modern studies have shown that YGY can enhance bone formation by promoting the proliferation of $\mathrm{OB}$ and inhibiting the formation of $\mathrm{OC}$, but the molecular mechanism has not been revealed in detail. Therefore, this study analyzed and discussed the potential mechanism with the help of network pharmacology and molecular docking, aiming to provide a scientific basis for the clinical application of YGY.

Based on network pharmacology, this study predicted 14 core effective ingredients including quercetin, ahumulene, cinnamaldehyde, benzoylaconine, catalpol, and 6 core targets of TNF- $a$, IL-1ß, IL-6, NF-kB, CTSK, CASP3. The results of molecular docking revealed that the core effective ingredients and core targets of YGY in the treatment of OP showed good binding activity, which verified the reliability of network pharmacology. The results of GO enrichment analysis showed that the biological process of YGY in the treatment of OP might be related to the regulation of inflammatory response and ossification. The representative pathways closely related to the above two biological processes were selected from the 
results of KEGG pathway enrichment analysis, including TNF signaling pathway, osteoclast differentiation and apoptosis. Figure 10 showed that the 6 core targets of TNF-a, IL-1 $\beta$, IL-6, NF-kB, CASP3 and CTSK involved in regulating these signal pathways might play important roles in the treatment of OP by YGY.

At present, a large number of studies have proven that the occurrence and development of OP is significantly associated with inflammation [25]. The proinflammatory cytokines TNF-a, IL-1 $\beta$ and IL-6 are important regulators of bone resorption [26]. TNF- $a$ can not only induce OB to express the key osteoclastogenic factor RANKL [27], but also stimulate OC precursors to express RANK [28]. The combination of RANKL and RANK will lead to OC differentiation and activation [29]. Similar to TNF-a, IL$1 \beta$ can promote RNAKL-induced OC formation by activating NF-kB [30-32]. The role of IL-6 in promoting STAT3-mediated RANKL expression is achieved by stimulating the glycoprotein gp130 receptor subunit on OB [33]. It can be seen that TNF- $a$, IL-1 $\beta$, and IL- 6 can all up-regulate the expression of RANKL. The interaction of RANKL and RANK can stimulate several factors including NFATc1 [34]. CTSK directly regulated by NFATC1 can degrade bone collagen, which is closely related to the bone resorption activity of OC $[35,36]$. In addition, TNF-a can inhibit the transcription of RUNX2, the main transcription factor for OB differentiation [37]. TNF- $\alpha$ also activates the activity of the apoptosis executive protein CASP3 in OB to induce OB apoptosis [38]. Therefore, the proinflammatory factors TNF-a, IL-1 $\beta$, and IL-6 can promote OC differentiation while inhibiting the differentiation and activity of $\mathrm{OB}$, ultimately leading to a negative balance of bone resorption and bone formation. Furthermore, the results of molecular docking showed that the binding energy of TNF, IL-1 $1 \beta, \mathrm{IL}-6$ and CTSK to more than 7 core effective ingredients were all less than -5.00 , which indicated that they were the key factors among the 6 core targets.

It can be seen from the results of molecular docking that quercetin, a-humulene, denudatine, cinnamaldehyde, benzoylaconine, benzoylmesaconine, benzoylhypaconine, mesaconitine and catalpol with binding energy to 3 or more core targets among TNF, IL-1 $1 \beta$, IL-6 and CTSK less than -5.00 were more important among the 14 core effective ingredients. Among them, quercetin, a-humulene, cinnamaldehyde, benzoylaconine and catalpol have been proven to exert regulatory effects on the 6 core targets to adjust the balance of bone metabolism. Quercetin has been proven to significantly reduce the expression of TNF- $a$, IL-1 $\beta$ and IL-6 in RAW264.7 cells induced by M-CSF, RANKL or LPS [39-41]. In addition, quercetin can inhibit OB apoptosis while inducing OC apoptosis by regulating several factors including CASP3 [42]. The systemic treatment with a-humulene can significantly inhibit the secretion of TNF-a and IL-1 $\beta$ [43]. ahumulene also inhibits the activation of NF-kB [44]. Both cinnamaldehyde and benzoylaconine can significantly inhibit the secretion of TNF- $\alpha$, IL-1 $\beta$, and IL- 6 by inhibiting multiple signal pathways, including the NF-kB signaling pathway [45-48]. Catalpol can inhibit the bone resorption of OC by effectively inhibiting the expression of CTSK [49]. Mesaconitine, benzoylmesaconine, benzoylhypaconine, denudatine and other core effective ingredients with low binding energy to the core targets may also have potential regulatory ability to the core targets and their roles in the treatment of OP by YGY cannot be underestimated. Obviously, the molecular mechanism of YGY in the treatment of OP is closely related to the regulation of proinflammatory factors. 
There are several limitations in this study. Some relevant targets and effective ingredients may be ignored due to the incomplete information of databases. In addition, even if the results of network pharmacology and molecular docking were combined, the exact mechanism of YGY in the treatment of OP still needed to be further verified through experiments. Nevertheless, the findings of this study provided direction and valuable enlightenment for follow-up research.

\section{Conclusions}

Based on network pharmacology and molecular docking, quercetin, a-humulene, denudatine, cinnamaldehyde, benzoylaconine, benzoylmesaconine, benzoylhypaconine, mesaconitine and catalpol were identified as critical effective ingredients, while TNF, IL-1 $\beta$, IL- 6 and CTSK were considered as the main targets. In conclusion, YGY is most likely to play a therapeutic effect on OP by inhibiting the activation and expression of the above targets through the above effective ingredients to regulate the signal pathway including TNF signaling pathway, osteoclast differentiation and apoptosis for improving the biological processes including inflammation response and ossification and eventually reducing bone resorption and promoting bone formation. This study systematically analyzed the material basis and potential mechanism of $Y G Y$ in the treatment of $O P$, which may provide a promising direction for further research on the exact mechanism of $Y G Y$ in the treatment of OP.

\section{Abbreviations}

YGY: Youguiyin; OP: osteoporosis; TCMSP: Traditional Chinese Medicine System Pharmacology Database and Analysis Platform; TTD: Therapeutic Target Database; OMIM: Online Mendelian Inheritance in Man; ADME: Absorption, Distribution, Metabolism and Excretion; DAVID: The Database for Annotation, Visualization and Integrated Discovery; PPI: Protein-protein interaction; GO: Gene ontology; KEGG: Kyoto Encyclopedia of Genes and Genomes

\section{Declarations}

Acknowledgements

Not applicable.

Authors' contributions

PY and ZL conceived and designed the study. PY and ZWL collected the data. PY analyzed the data and wrote the article. ZL and QLP arevised the manuscript. All authors read and approved the fnal manuscript.

Funding 
This study was supported by the grant from the National Natural Science Foundation for the Youth of China (NO.82004247), the Exploration Project of Natural Science Foundation of Zhejiang Province (NO.LQ21H270002), the Young and Middle-aged Scientific Research Innovation Project of Zhejiang University of Traditional Chinese Medicine (NO.2019ZR16).

Availability of data and materials

The datasets used and/or analysed during the current study are available from the corresponding author on reasonable request.

Ethics approval and consent to participate

Not applicable.

Consent for publication

Not applicable.

Competing interests

The authors declare no competing interests regarding the publication of this manuscript.

\section{References}

1. Yu F, Xia W. The epidemiology of osteoporosis, associated fragility fractures, and management gap in China. Arch Osteoporos. 2019;14(1):32.

2. Gong R, Ren S, Chen M, et al. Bioinformatics Analysis Reveals the Altered Gene Expression of Patients with Postmenopausal Osteoporosis Using Liuweidihuang Pills Treatment [published correction appears in Biomed Res Int. 2019 Nov 20;2019:6897187]. Biomed Res Int. 2019;2019:1907906.

3. Si L, Winzenberg TM, Jiang Q, Chen M, Palmer AJ. Projection of osteoporosis-related fractures and costs in China: 2010-2050. Osteoporos Int. 2015;26(7):1929-37.

4. Muruganandan S, Govindarajan R, Sinal CJ. Bone Marrow Adipose Tissue and Skeletal Health. Curr Osteoporos Rep. 2018;16(4):434-42.

5. Shang Q, Zhao W, Shen G, et al. Jingui Shenqi Pills Regulate Bone-Fat Balance in Murine Ovariectomy-Induced Osteoporosis with Kidney Yang Deficiency. Evid Based Complement Alternat Med. 2020;2020:1517596.

6. He Y, Bao YT, Chen HS, et al. The Effect of Shen Qi Wan Medicated Serum on NRK-52E Cells Proliferation and Migration by Targeting Aquaporin 1 (AQP1). Med Sci Monit. 2020;26:e922943.

7. Wu CL, Li TY, Wu JS, et al. The effect of Youguiyin on the differentiation and function of osteoclasts in vitro. Chinese Archives of Traditional Chinese Medicine. 2010;28(02):304-7. (in Chinese with English abstract). 
8. Qi XY, Liu H, Bi DD, et al. Combined administration on You-Gui Yin and low-dose Raloxifene partially attenuates the bone loss in ovariectomized mice through the proliferation and osteogenic differentiation of bone marrow stromal cells. Phytomedicine. 2019;53:286-93.

9. Yang A, Yu C, You F, He C, Li Z. Mechanisms of Zuogui Pill in Treating Osteoporosis: Perspective from Bone Marrow Mesenchymal Stem Cells. Evid Based Complement Alternat Med. 2018;2018:3717391.

10. Ren B, Tan L, Xiong Y, et al. Integrated Analysis of the Mechanisms of Da-Chai-Hu Decoction in Type 2 Diabetes Mellitus by a Network Pharmacology Approach. Evid Based Complement Alternat Med. 2020;2020:9768414.

11. Ru J, Li P, Wang J, et al. TCMSP: a database of systems pharmacology for drug discovery from herbal medicines. J Cheminform. 2014;6:13.

12. Daina A, Michielin O, Zoete V. SwissTargetPrediction: updated data and new features for efficient prediction of protein targets of small molecules. Nucleic Acids Res. 2019;47(W1):W357-64.

13. Li YH, Yu CY, Li XX, et al. Therapeutic target database update 2018: enriched resource for facilitating bench-to-clinic research of targeted therapeutics. Nucleic Acids Res. 2018;46(D1):D1121-7.

14. Hamosh A, Scott AF, Amberger JS, Bocchini CA, McKusick VA. Online Mendelian Inheritance in Man (OMIM), a knowledgebase of human genes and genetic disorders. Nucleic Acids Res. 2005;33(Database issue):D514-7.

15. Huang da W, Sherman BT, Lempicki RA. Systematic and integrative analysis of large gene lists using DAVID bioinformatics resources. Nat Protoc. 2009;4(1):44-57.

16. Szklarczyk D, Gable AL, Lyon D, et al. STRING v11: protein-protein association networks with increased coverage, supporting functional discovery in genome-wide experimental datasets. Nucleic Acids Res. 2019;47(D1):D607-13.

17. Zhou Y, Zhou B, Pache L, et al. Metascape provides a biologist-oriented resource for the analysis of systems-level datasets. Nat Commun. 2019;10(1):1523.

18. Burley SK, Bhikadiya C, Bi C, et al. RCSB Protein Data Bank: powerful new tools for exploring 3D structures of biological macromolecules for basic and applied research and education in fundamental biology, biomedicine, biotechnology, bioengineering and energy sciences. Nucleic Acids Res. 2021;49(D1):D437-51.

19. Kim S, Chen J, Cheng T, et al. PubChem in 2021: new data content and improved web interfaces. Nucleic Acids Res. 2021;49(D1):D1388-95.

20. Hsin KY, Ghosh S, Kitano H. Combining machine learning systems and multiple docking simulation packages to improve docking prediction reliability for network pharmacology. PLoS One. 2013;8(12):e83922.

21. Shen Y, Jia LN, Honma N, Hosono T, Ariga T, Seki T. Beneficial effects of cinnamon on the metabolic syndrome, inflammation, and pain, and mechanisms underlying these effects - a review. J Tradit Complement Med. 2012;2(1):27-32.

22. Li M, He J, Jiang LL, et al. The anti-arthritic effects of Aconitum vilmorinianum, a folk herbal medicine in Southwestern China. J Ethnopharmacol. 2013;147(1):122-7. 
23. Zhang RX, Li MX, Jia ZP. Rehmannia glutinosa: review of botany, chemistry and pharmacology. J Ethnopharmacol. 2008;117(2):199-214.

24. Viljakainen HT, Koistinen HA, Tervahartiala T, Sorsa T, Andersson S, Mäkitie O. Metabolic milieu associates with impaired skeletal characteristics in obesity. PLoS One. 2017;12(6):e0179660.

25. Brincat SD, Borg M, Camilleri G, Calleja-Agius J. The role of cytokines in postmenopausal osteoporosis. Minerva Ginecol. 2014;66(4):391-407.

26. McLean RR. Proinflammatory cytokines and osteoporosis. Curr Osteoporos Rep. 2009;7(4):134-9.

27. Nakao A, Fukushima H, Kajiya H, Ozeki S, Okabe K. RANKL-stimulated TNFalpha production in osteoclast precursor cells promotes osteoclastogenesis by modulating RANK signaling pathways. Biochem Biophys Res Commun. 2007;357(4):945-50.

28. Komine M, Kukita A, Kukita T, Ogata Y, Hotokebuchi T, Kohashi O. Tumor necrosis factor-alpha cooperates with receptor activator of nuclear factor kappaB ligand in generation of osteoclasts in stromal cell-depleted rat bone marrow cell culture. Bone. 2001;28(5):474-83.

29. Lu X, Gilbert L, He X, Rubin J, Nanes MS. Transcriptional regulation of the osterix (Osx, Sp7) promoter by tumor necrosis factor identifies disparate effects of mitogen-activated protein kinase and NF kappa B pathways. J Biol Chem. 2006;281(10):6297-306.

30. Zha L, He L, Liang Y, et al. TNF-a contributes to postmenopausal osteoporosis by synergistically promoting RANKL-induced osteoclast formation. Biomed Pharmacother. 2018;102:369-74.

31. Jimi E, Nakamura I, Ikebe T, Akiyama S, Takahashi N, Suda T. Activation of NF-kappaB is involved in the survival of osteoclasts promoted by interleukin-1. J Biol Chem. 1998;273(15):8799-805.

32. Son HS, Lee J, Lee HI, et al. Benzydamine inhibits osteoclast differentiation and bone resorption via down-regulation of interleukin-1 $\beta$ expression. Acta Pharm Sin B. 2020;10(3):462-74.

33. O'Brien CA, Lin SC, Bellido T, Manolagas SC. Expression levels of gp130 in bone marrow stromal cells determine the magnitude of osteoclastogenic signals generated by IL-6-type cytokines. J Cell Biochem. 2000;79(4):532-41.

34. Asagiri M, Sato K, Usami T, et al. Autoamplification of NFATc1 expression determines its essential role in bone homeostasis. J Exp Med. 2005;202(9):1261-9.

35. Song I, Kim JH, Kim K, Jin HM, Youn BU, Kim N. Regulatory mechanism of NFATc1 in RANKL-induced osteoclast activation. FEBS Lett. 2009;583(14):2435-40.

36. Stone JA, McCrea JB, Witter R, Zajic S, Stoch SA. Clinical and translational pharmacology of the cathepsin K inhibitor odanacatib studied for osteoporosis. Br J Clin Pharmacol. 2019;85(6):1072-83.

37. Gilbert L, He X, Farmer P, et al. Inhibition of osteoblast differentiation by tumor necrosis factor-alpha. Endocrinology. 2000;141(11):3956-64.

38. Bradford PG, Gerace KV, Roland RL, Chrzan BG. Estrogen regulation of apoptosis in osteoblasts. Physiol Behav. 2010;99(2):181-5.

39. Tang J, Diao P, Shu X, Li L, Xiong L. Quercetin and Quercitrin Attenuates the Inflammatory Response and Oxidative Stress in LPS-Induced RAW264.7 Cells: In Vitro Assessment and a Theoretical Model. 
Biomed Res Int. 2019;2019:7039802.

40. Zhang L, Tian Z, Li W, Wang X, Man Z, Sun S. Inhibitory effect of quercetin on titanium particleinduced endoplasmic reticulum stress (ERS)-related apoptosis and in vivoosteolysis. Biosci Rep. 2017;37(4):BSR20170961.

41. Ge YW, Feng K, Liu XL, et al. Quercetin inhibits macrophage polarization through the $p-38 a / \beta$ signalling pathway and regulates OPG/RANKL balance in a mouse skull model. J Cell Mol Med. 2020;24(5):3203-16.

42. Wong SK, Chin KY, Ima-Nirwana S. Quercetin as an Agent for Protecting the Bone: A Review of the Current Evidence. Int J Mol Sci. 2020;21(17):6448.

43. Fernandes ES, Passos GF, Medeiros R, et al. Anti-inflammatory effects of compounds alphahumulene and (-)-trans-caryophyllene isolated from the essential oil of Cordia verbenacea. Eur $\mathrm{J}$ Pharmacol. 2007;569(3):228-36.

44. Medeiros R, Passos GF, Vitor CE, et al. Effect of two active compounds obtained from the essential oil of Cordia verbenacea on the acute inflammatory responses elicited by LPS in the rat paw. Br J Pharmacol. 2007;151(5):618-27.

45. Chen P, Ruan A, Zhou J, et al. Cinnamic Aldehyde Inhibits Lipopolysaccharide-Induced Chondrocyte Inflammation and Reduces Cartilage Degeneration by Blocking the Nuclear Factor-Kappa B Signaling Pathway. Front Pharmacol. 2020;11:949.

46. Li X, Wang Y. Cinnamaldehyde Attenuates the Progression of Rheumatoid Arthritis through DownRegulation of PI3K/AKT Signaling Pathway. Inflammation. 2020;43(5):1729-41.

47. Yu HH, Li M, Li YB, et al. Benzoylaconitine Inhibits Production of IL-6 and IL-8 via MAPK, Akt, NF-KB Signaling in IL-1 $\beta$-Induced Human Synovial Cells. Biol Pharm Bull. 2020;43(2):334-9.

48. Gai W, Hao X, Zhao J, et al. Delivery of benzoylaconitine using biodegradable nanoparticles to suppress inflammation via regulating NF-KB signaling. Colloids Surf B Biointerfaces. 2020;191:110980.

49. Meng J, Zhang W, Wang C, et al. Catalpol suppresses osteoclastogenesis and attenuates osteoclastderived bone resorption by modulating PTEN activity. Biochem Pharmacol. 2020;171:113715.

\section{Figures}




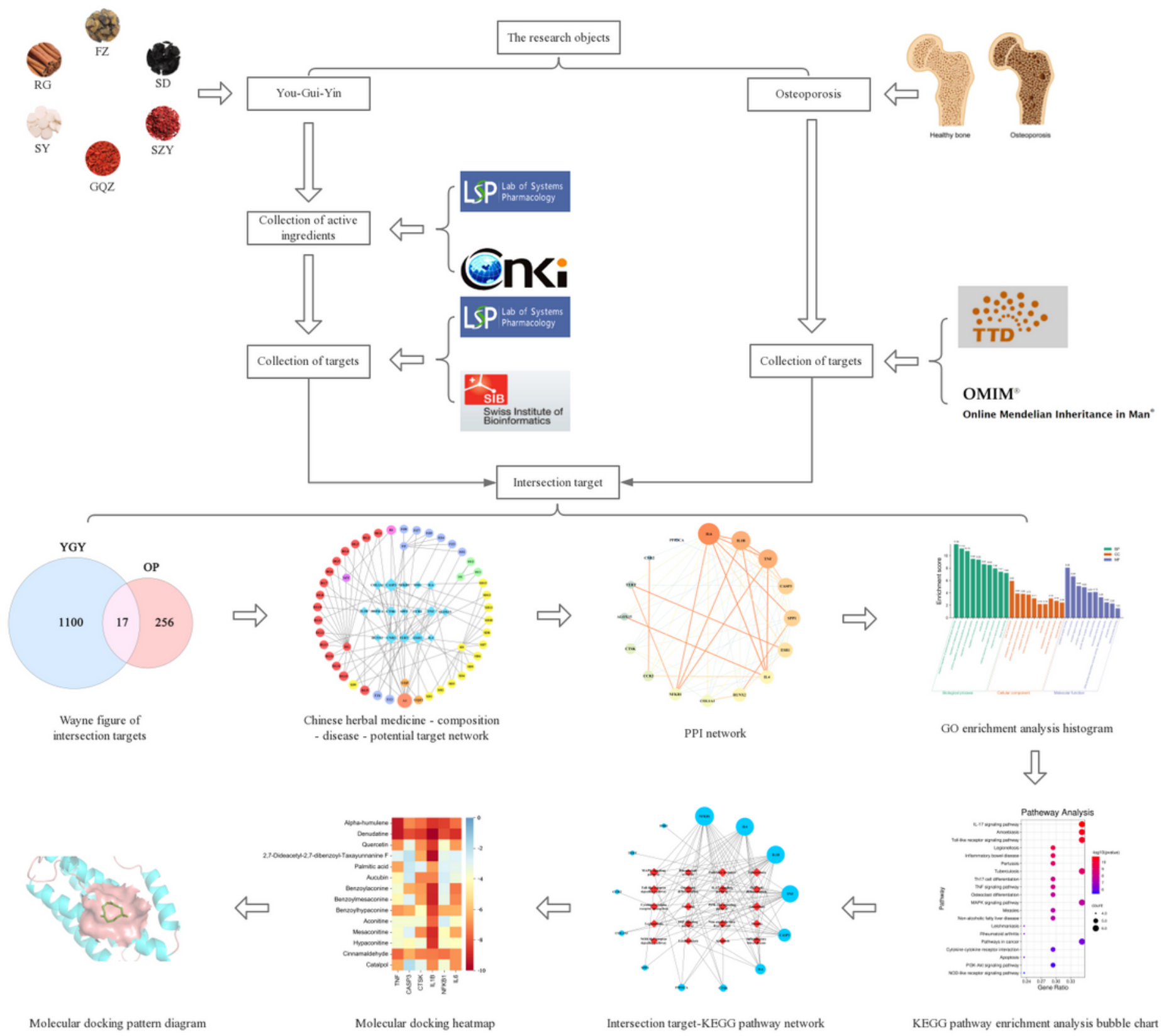

\section{Figure 1}

The schematic diagram of this study based on network pharmacology and molecular docking for exploring the potential mechanism of YGY against OP 


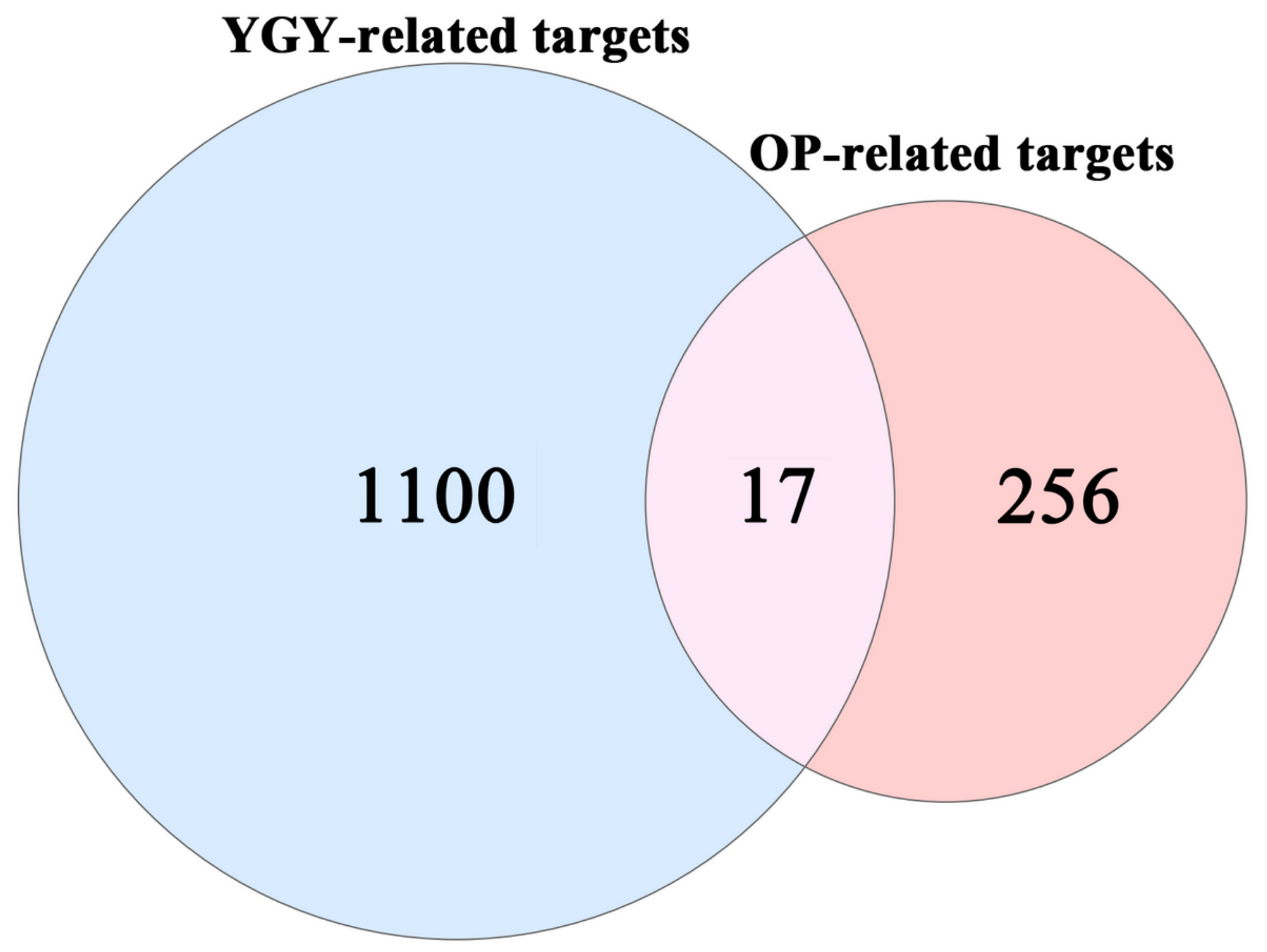

Figure 2

The venn diagram for YGY-related targets and OP-related targets. The intersection targets mean the potential targets of $Y G Y$ in the treatment of $O P$ 


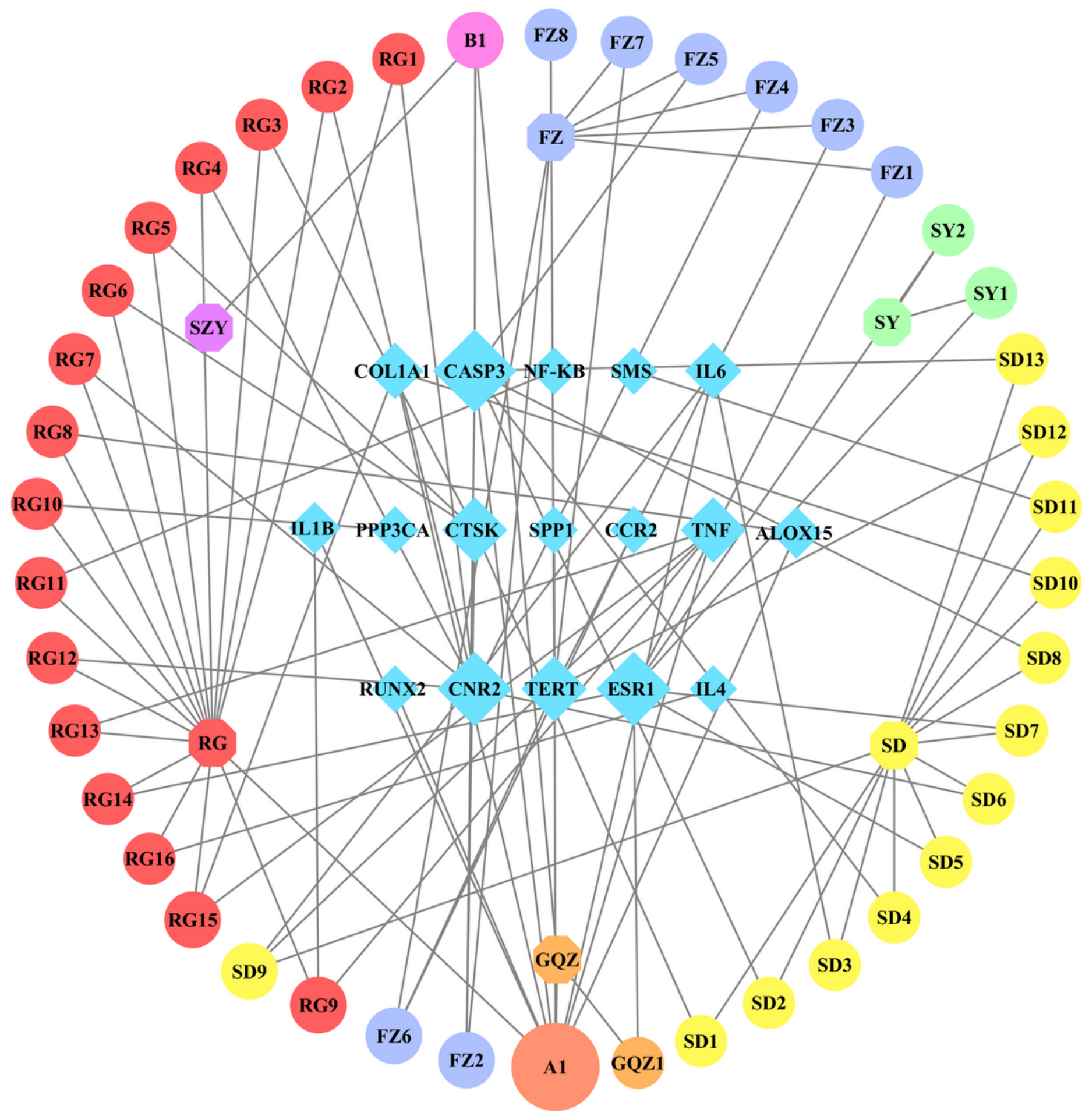

Figure 3

The Chinese medicine-compound-OP-target network of YGY in the treatment of OP 


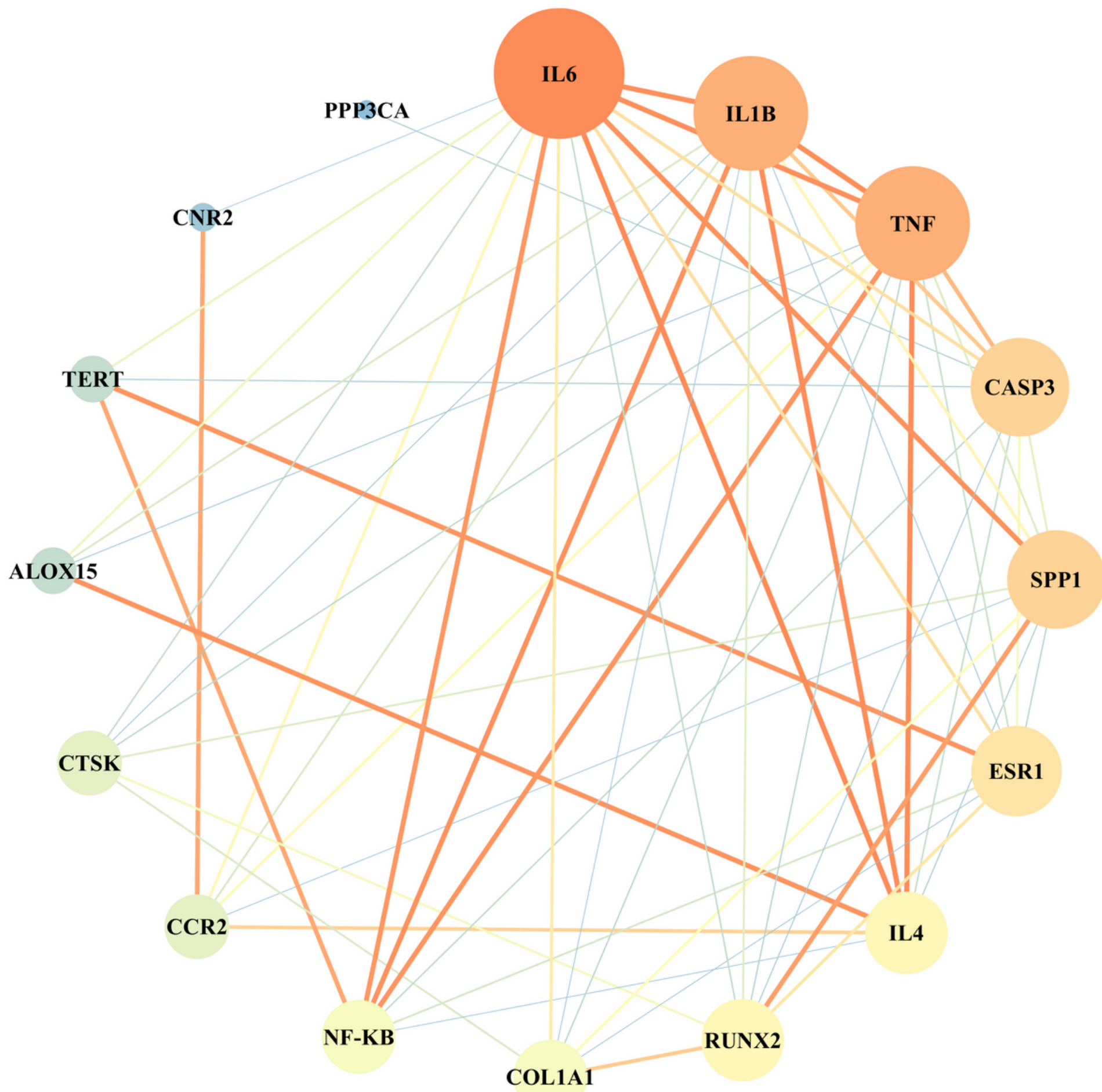

Figure 4

The PPI network of the intersection targets 


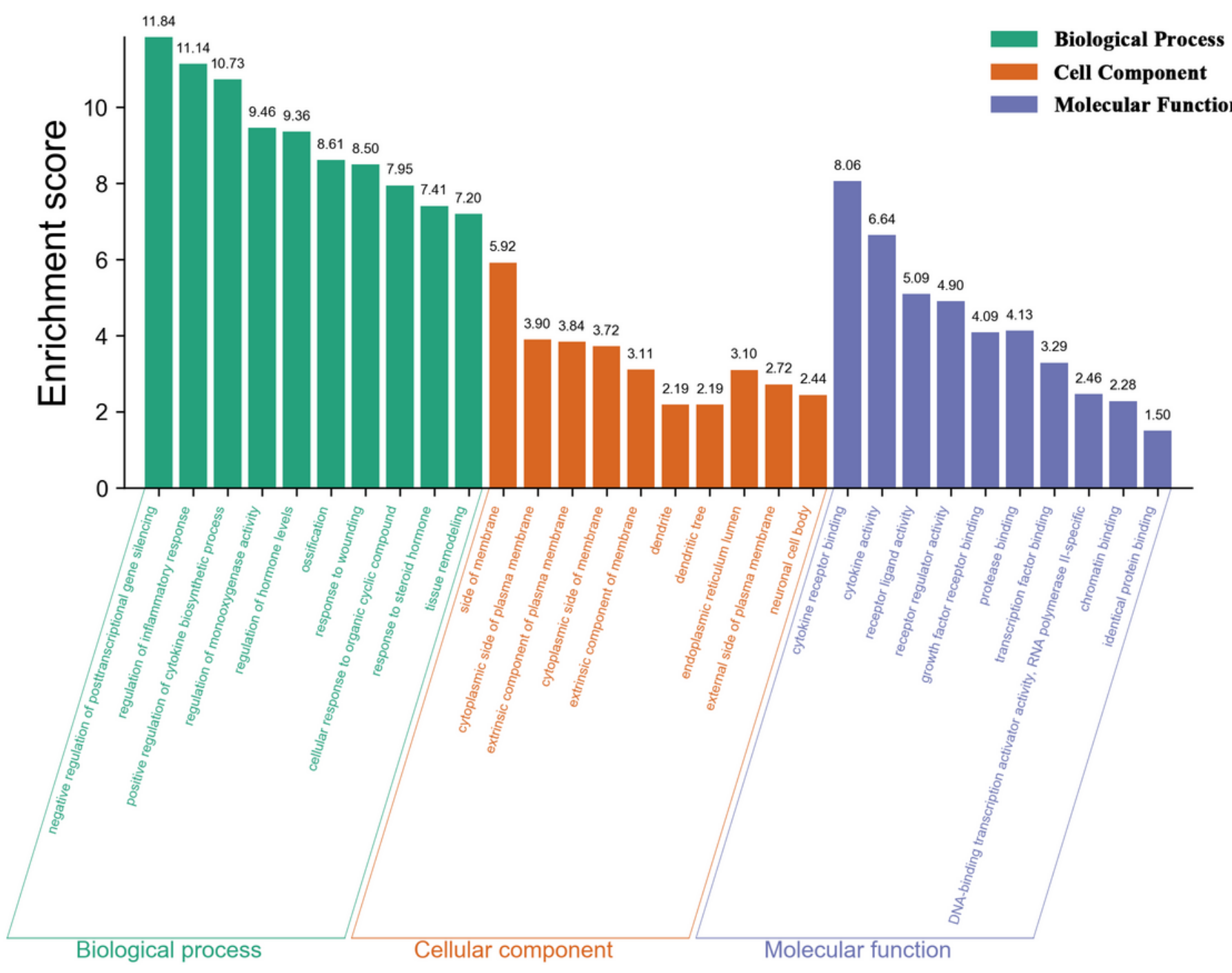

Figure 5

GO enrichment analysis of the intersection targets 


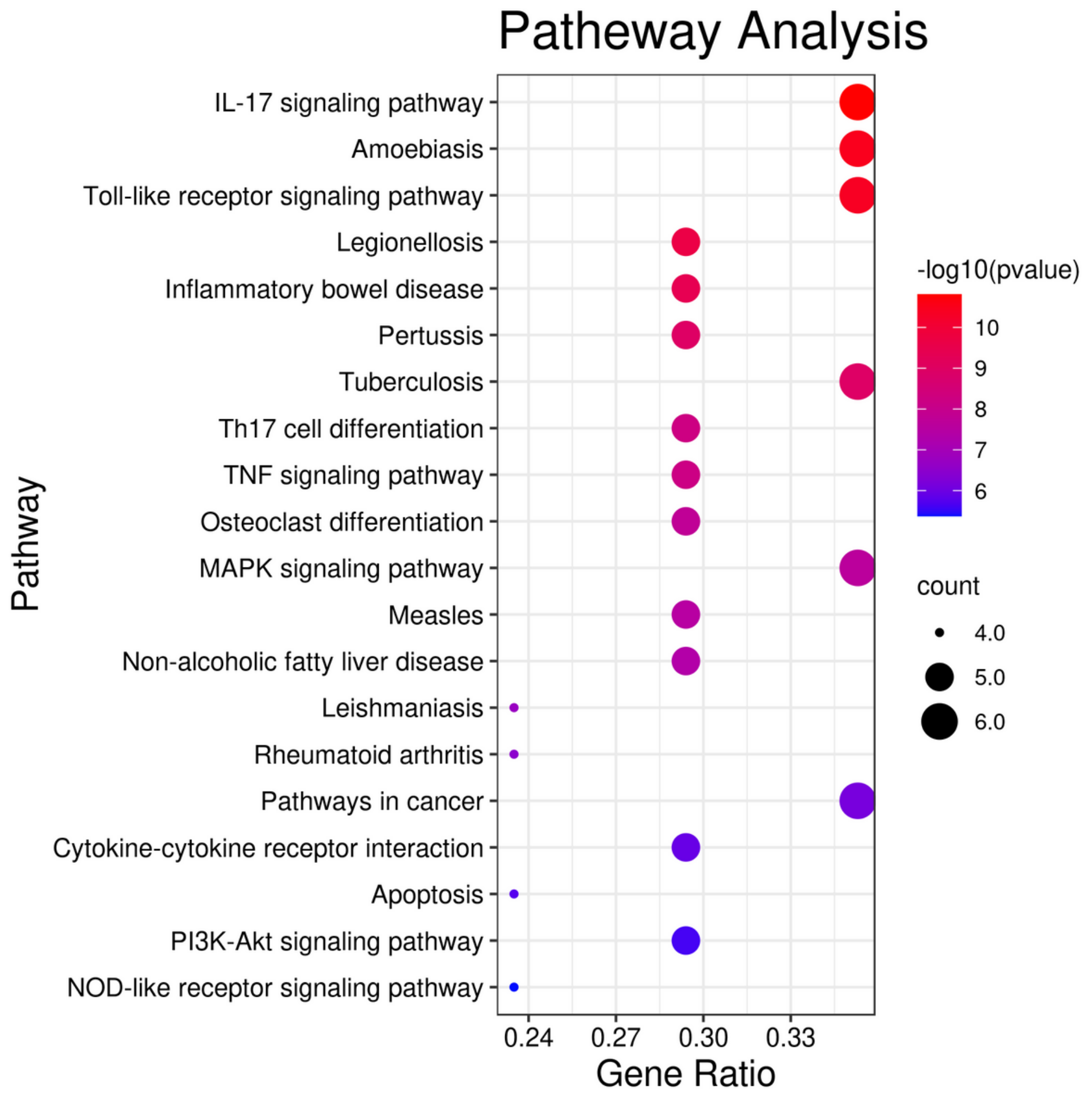

Figure 6

KEGG pathway enrichment analysis of the intersection targets 


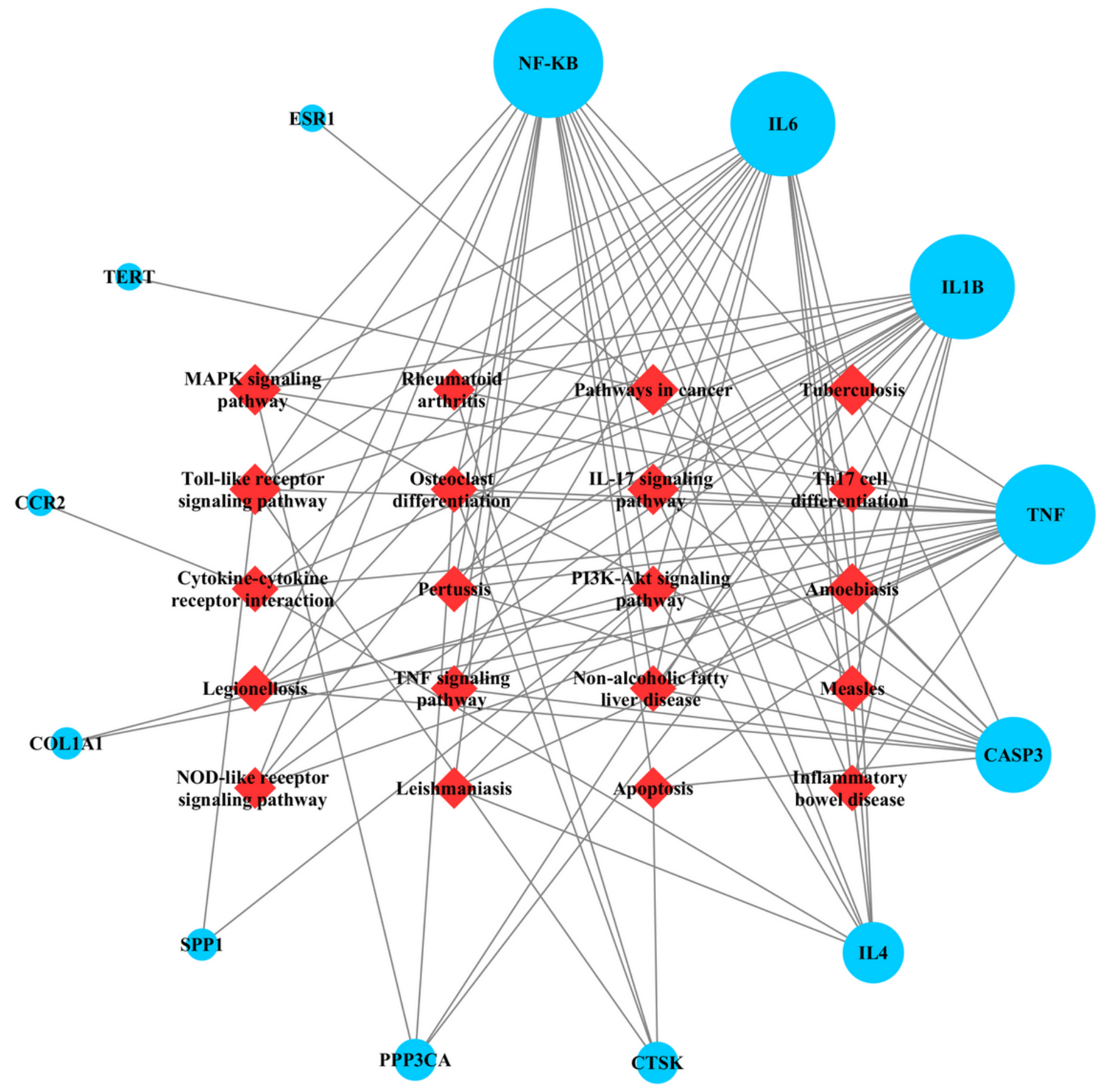

Figure 7

The intersection target-signal pathway network of YGY in the treatment of OP 


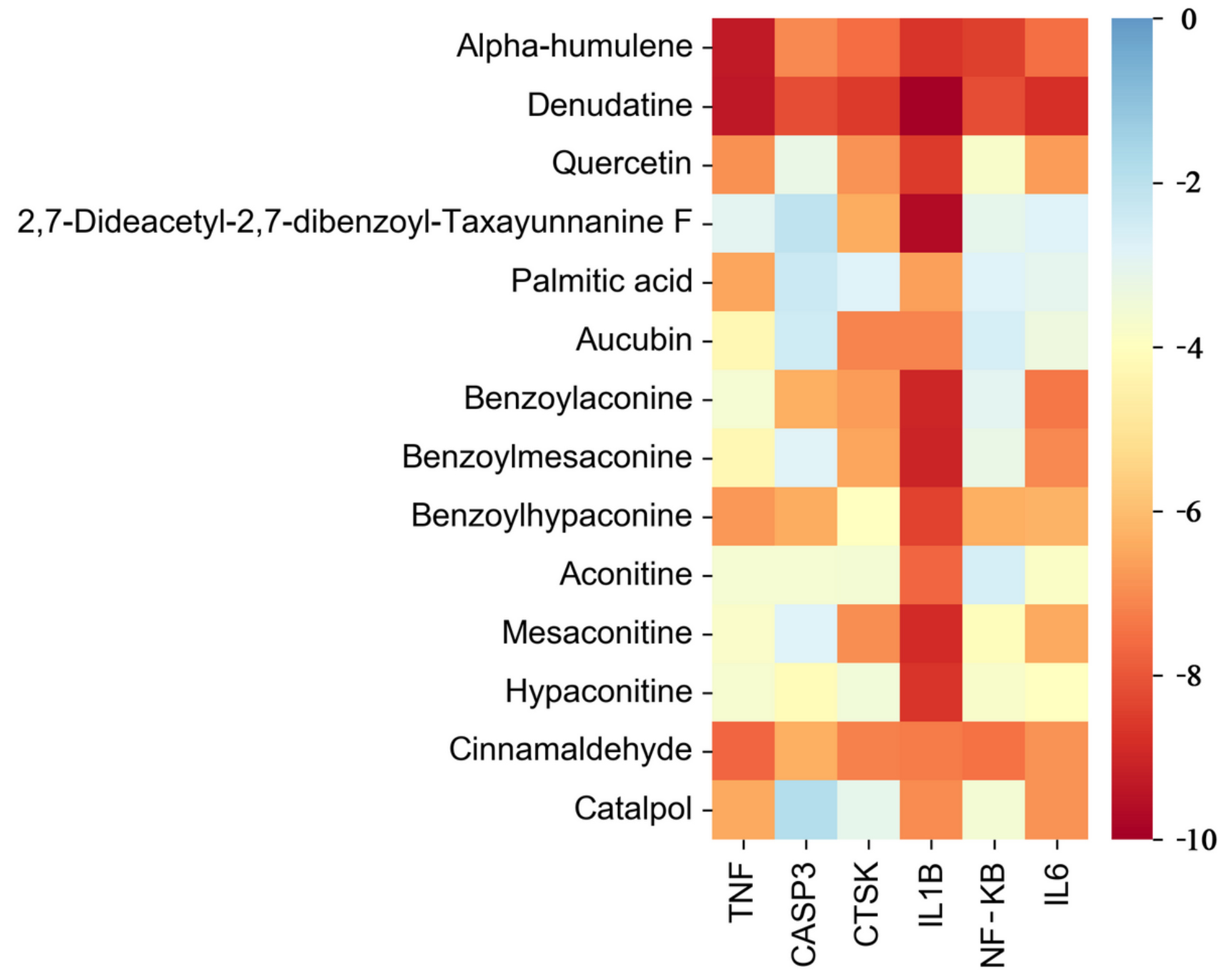

Figure 8

Heat maps of the binding energy for the core targets combined with the effective ingredients 


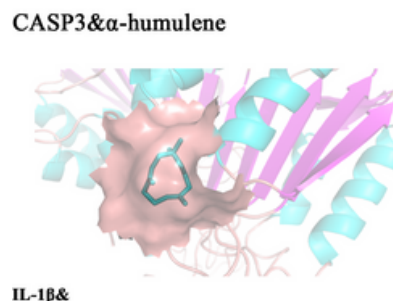

2,7-Dideacetyl-2,7-dibenzoyl-Taxayunnanine F

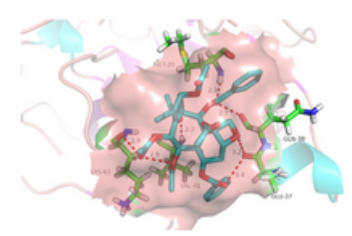

NF-KB\& $\alpha$-humulene

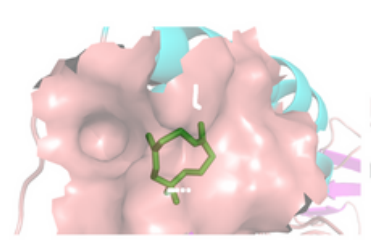

CASP3\&denudatine

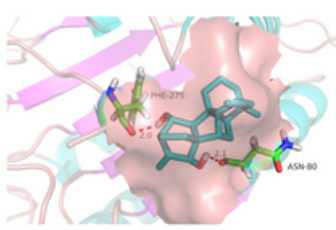

IL-1 $\beta \&$ benzoylaconine

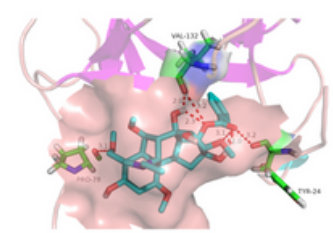

NF-KB\&denudatine

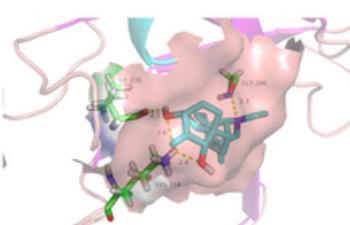

CTSK\& $\alpha$-humulene

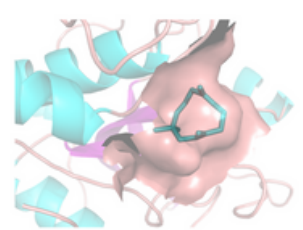

IL-1 $\beta \&$ denudatine (1)

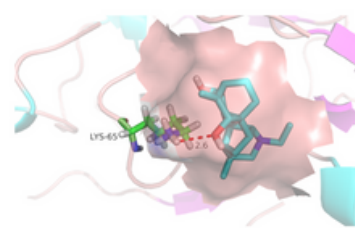

TNF\& $\alpha$-humulene

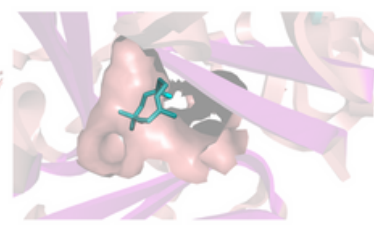

CTSK\&denudatine

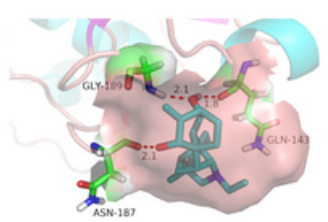

IL-1 $\beta \&$ denudatine (2)

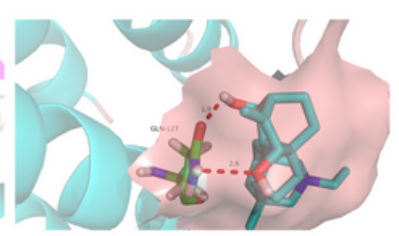

TNF\&cinnamaldehyde

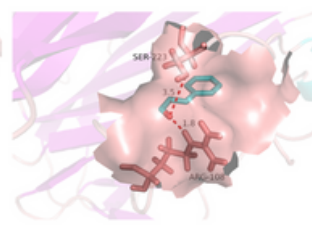

IL-1 $\beta \&$ benzoylmesaconine

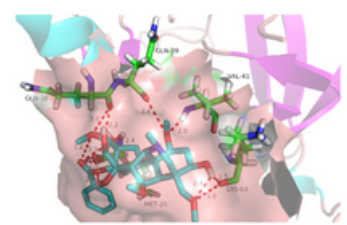

IL-1 $\beta \& \alpha$-humulene

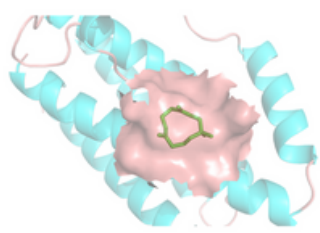

TNF\&denudatine

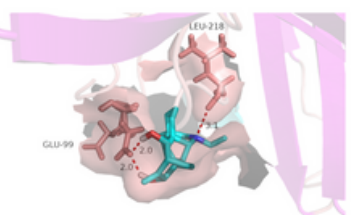

\section{Figure 9}

Molecular docking of the effective ingredients with core targets

TNF signaling pathway

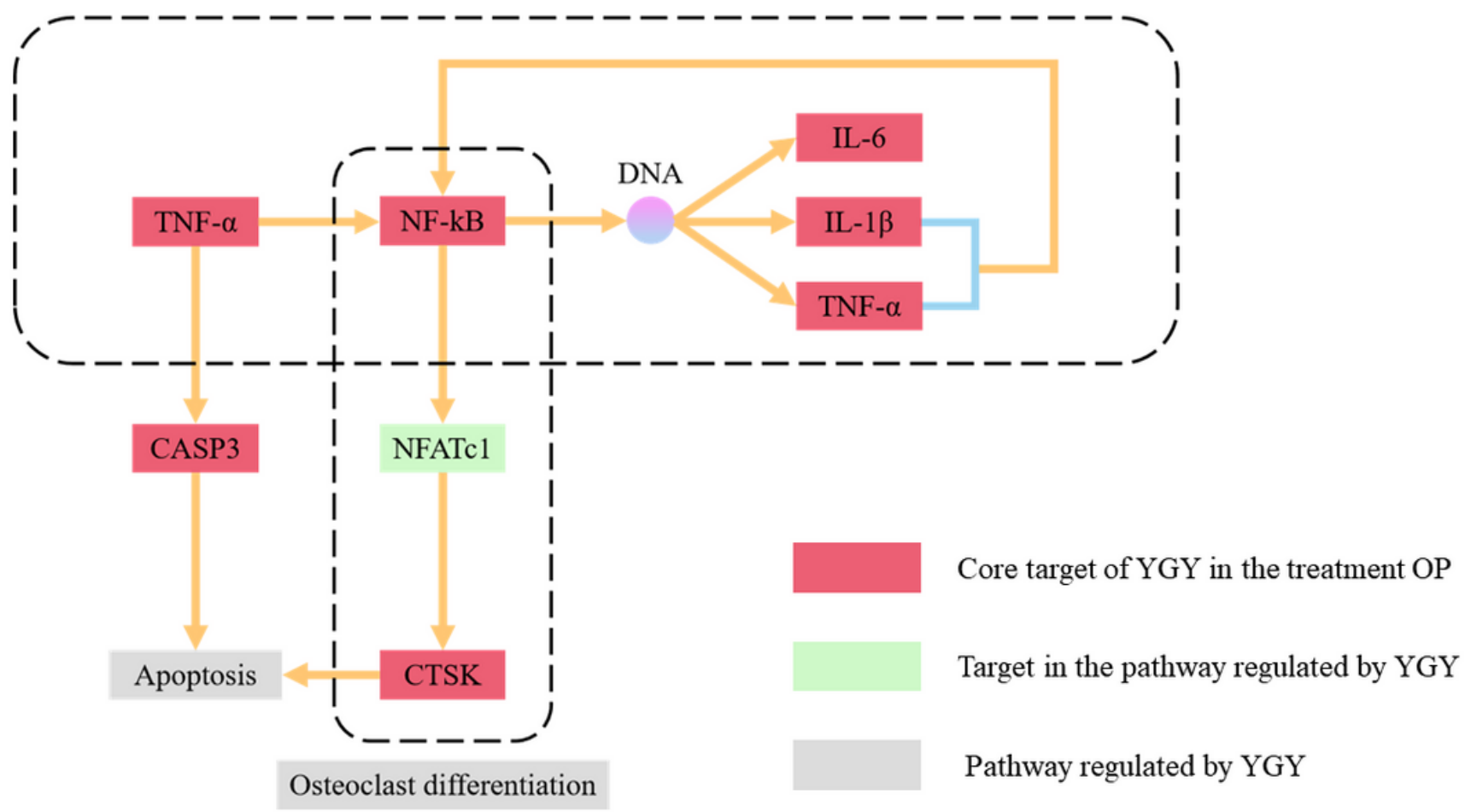

\section{Figure 10}

The representative signal pathways of OP regulated by the core target of YGY 